\title{
Research on the method of the Test and Evaluate (T\&E) of Complex Simulation System
}

\author{
Li Liu ${ }^{1}$ a, Xu Chang $\mathrm{Li}^{1}$, Kun Wang ${ }^{2}$, Xiao Min Zhang ${ }^{2}$, Hua Xiao ${ }^{2}$ \\ 1.Air Force Engineering University, Xi'an China \\ 2.Chinese Flight Test Establishment,Xi'an China
}

\begin{abstract}
With the development of science and evolution of society, the structure of a system becomes more and more complicated. There is an increasing need for the research, development and application of complex systems. Based on the brief introduction to the significance of T\&E, the relationship between T\&E and development status in the domestic and overseas, expounding the part of the establishment of the theory and methodology of T\&E at the technical and methodological level, which offers references for the further development of the test and evaluation method and technology of T\&E of complex systems.
\end{abstract}

Keywords- complex systems; Modeling and Simulation (M\&S); Test and Evaluation (T\&E)

\section{INTRODUCTION}

The simulation is the whole process to predict the future, aid for design, research and develop products and do scientific research with model operation. It is different from the theoretical and experimental research, but based on the scientific model operation and engineering activities. The establishment of a credible simulation system that meets the application target is the basic requirement of the user, detection and evaluation of operation data is necessary and a fundamental guarantee to meet this requirement. Therefore, the simulation must answer and prove the following three major issues seriously: (1) From the source, if the model correctly to describe the actual system (process) features and characteristics or not? (2) in operation, if the simulation effectively reflect data, character and behavior or not? (3) Analysis, if the simulation result really realize the application target and user requirement or not? These problems must be solved through the test and evaluation of complex simulation system.

\section{THE SIGNIFICANCE AND ROLE OF COMPLEX SIMULATION SYSTEM T\&E}

$\mathrm{T} \& \mathrm{E}$ is the necessary work processes and technologies to ensure that its complex simulation system is special credibility or credibility, its significance and function is very important, the main reflect are the following several aspects:
(1) It can effectively improve the correctness of the simulation results; strengthen the confidence in application of the simulation system;

(2) It can reduce the risk of the building and application of simulation system, especially to avoid disastrous risk, to shorten the development cycle, improves the efficiency of development;

(3) It may reduce the total investment of the simulation system, and improve the effectiveness and efficiency;

(4) It is advantageous to the comprehensive analysis of the problems of the scientific research;

(5) It expands the scope of the simulation system, to improve the reusability of the simulation system;

6) It can promote simulation system of total quality management; improve the management level and efficiency (reference to figure1).

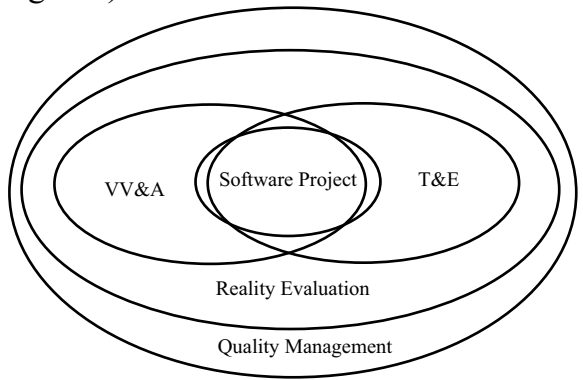

Fig1. The relationships among T\&E, Reality Evaluation, VV\&A and Quality Management of Complex Simulation System

${ }^{a}$ Corresponding author: 11200010@126.com 


\section{THE DEVELOPMENT TENDENCY IN CHINA AND ABROAD}

Abroad, especially in the United States, the researches about complex simulation system $\mathrm{T} \& \mathrm{E}$ are treated seriously and always walk forward the whole world. In the last century the America defense systems Management College drawn up "T\&E management guide". The modeling and simulation of the US department of defense office (DEMO) lists 76 kinds of software testing and system evaluation methods and 18 kinds of statistical techniques, which can be used for the simulation and verification of $\mathrm{V} \& \mathrm{~V}$. In recent years computer aided $\mathrm{V} \& \mathrm{~V}$ and T\&E tools have been developed, such as FVT, EE, Osman, Robin2002, VV\&A Turbo tools etc. Currently $\mathrm{T} \& \mathrm{E}$ is of integration, automation, intelligence and commercial.

T\&E has been development and application history in china earlier, especially in the field of aviation and space flight, in the 1980s the research application began. Many units (such as the Chinese flight test institute, 618, 623, etc. ) have already adopted the advanced spectrum analyzer and programmable frequency characteristic to test instrument for real-time simulation system by running T\&E orders. Recently, the intelligence and simulation in this field has become a hot spot.

\section{THEORY AND METHOD SYSTEM OF THE COMPLEX SIMULATION SYSTEM'S TEST AND EVALUATION}

It is necessary to build corresponding theory and method system for any scientific research, and the complex simulation system's T\&E is no exception. Research and perfecting theory and method system of the complex simulation system's T\&E constantly is crucial to meet the new needs of the complex simulation system's T\&E. Since Professor Osman Balci came up with the concept of VV\&T, the technology and application of T\&E have been developing constantly, and it has form a thorough theory and method system today. Author presents a high-level overview of the theory and method which is used by predecessors in the research of T\&E, gives the framework of the theory and method system of the complex simulation system's T\&E (as show in Figure2).

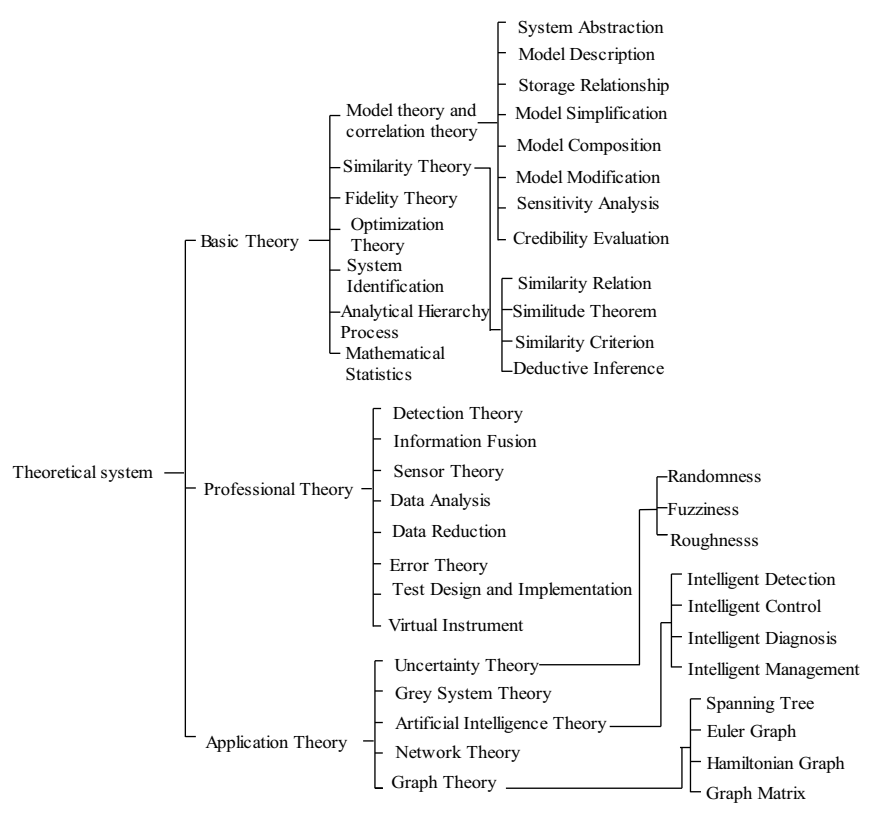

(a) Theoretical system

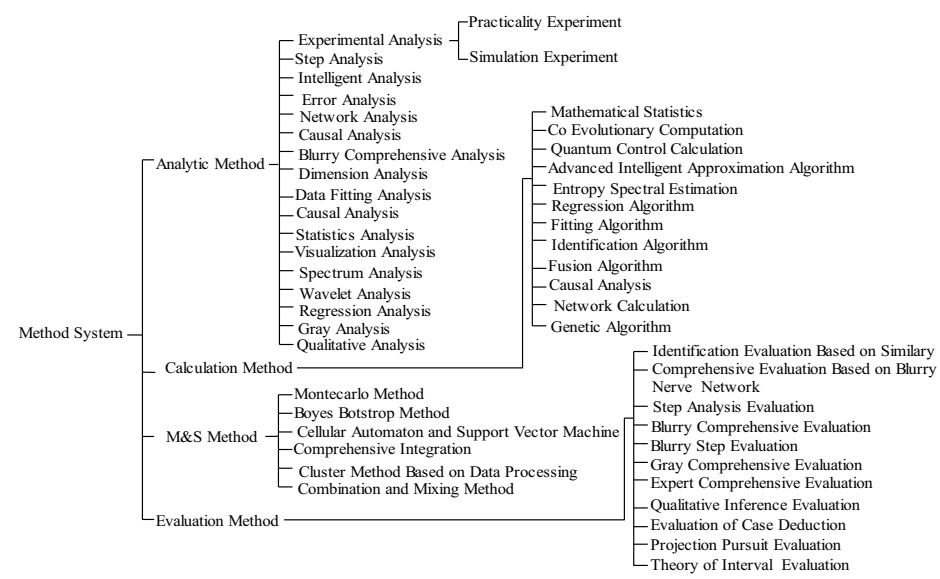

(b) Method System

Fig2 T\&E theory and method system structure developed/moved by complex simulation system

At present, the T\&E method for development /operation of the complex simulation system has been not a few, but for large complex simulation system $\mathrm{T} \& \mathrm{E}$ tend to work hard. As a result, it is very necessary to study the suitable T\&E method of his kind of simulation system. At present, a T\&E engineering method based on similarity theory and the identification technology has emerged, it is from the system and the similarity of concept model, using system 
identification techniques, can be directly by the similarity to determine the credibility of the complex simulation system. Practice has proved that this is a new credibility evaluation method with excellent operation. In addition, the identification evaluation method based on fuzzy neural network is a kind of combined modeling method, also suitable for large complex simulation system, and make the intelligent control of complex simulation system possible.

\section{Conclusion}

It is very difficult to monitor and evaluate the complex simulation system mainly because of the uncertainty (including randomness, fuzziness, and roughness, etc) in operation, serious nonlinear and the diversity of information and so on. For an efficient monitoring and evaluation, to provide effective and correct basis of the credibility, it is crucial to use artificial intelligence technology and computer aided analysis.

\section{REFERENCES}

[1] Xingtang Liu, Li Liu. Simulation Science Technique and Engineering. Beijing: Sciences Publishing House, 2013.03

[2]Xingtang Liu, Bingcheng Liang. The Theory, Method \& Technique for Complex System Modeling. Beijing : Sciences Publishing House, 2011.04

[3]Xingtang Liu, Li Liu. Modern Modeling and Simulation Technology. Northwestern Polytechnical University Publishing House. 2011.03

[4]Jianjun Yang. Scientific Research Method Introduction. Beijing: National Defense Industry Press, 2006.01

[5]Henglin Wang, Jianguo Cao. Simulation System Design and Application. Sciences Publishing House., 2003. 